\title{
Evaluation of terrestrial and airborne gravity data over Antarctica - a generic approach
}

https://doi.org/10.1515/jogs-2019-0004

Received January 25, 2019; accepted April 30, 2019

\begin{abstract}
The AntGrav project, funded by the German Research Foundation (DFG) has the main objective to homogenize and optimize Antarctic gravity field information. Within this project an evaluation procedure is needed to inspect all different kind of gravity field surveys available in Antarctica. In this paper a suitable methodology is proposed.

We present an approach for fast 3D gravity point data reduction in different spectral bands. This is achieved through pre-calculating a fine 3D mesh of synthesized gravity functionals over the entirety of the Antarctic continent, for which two different global models are used: the combined satellite model GOCO05s for the longwavelength part, and the topographic model Earth2014 for the shorter wavelengths. To maximize the applicability separate meshes are calculated for different spectral bands in order to specifically reduce a certain band or a selected combination. All meshes are calculated for gravity anomalies as well as gravity disturbances. Utilizing these meshes, synthesized gravity data at arbitrary positions is computed by conventional 3D interpolation methods (e.g. linear, cubic or spline).

It is shown that the applied approach can reach a worstcase interpolation error of less than $1 \mathrm{mGal}$. Evaluation results are presented for the AntGG grid and exemplary for the in-situ measurements of the AGAP and BAS-LAND campaigns. While general properties, large-scale errors and systematic effects can usually be detected, small-scale errors (e.g. of single points) are mostly untraceable due to the uncertainties within the topographic model.
\end{abstract}

Keywords: Antarctica, combined gravity field modelling, fast synthesis method, global geopotential model, gravity field

\footnotetext{
*Corresponding Author: P. Zingerle: Institute of Astronomical and Physical Geodesy, Technical University of Munich, Germany, E-mail: zingerle@tum.de

M. Scheinert, T. Schaller: Institut für Planetare Geodäsie, Technische Universität Dresden, Germany
}

\section{Introduction}

An accurate and consistent knowledge of the gravity field in Antarctica is crucial for many geophysical applications and thus also for a better understanding of the geological structures of the continent. In order to achieve this objective, Scheinert et al. (2016) presented a first continentwide collection of gravity field observations within the AntGG grid, containing a majority of all accessible measurements. In concrete, more than 13 million observations collected over the past decades covering about $73 \%$ of the continent are included in the AntGG processing. These measurement campaigns have been performed by many different countries using different measurement techniques and analysis methods. Thus, the entire collection of gravity observations is largely heterogeneous regarding:

- Spatial distribution: different campaigns may show different spatial distribution patterns. As an example, airborne campaigns usually have a high resolution in the along-track direction, while the cross-track resolution mostly depends on the airborne campaign goals (e.g. grid-vs. star-shaped). Terrestrial observations on the other hand may show no clear pattern at all including larger data gaps or even having just a onedimensional extent along a profile line.

- Observation type: as the earliest campaigns date back to a time before global navigation satellite systems became available, the observed data had to be processed in terms of gravity anomalies using physical heights, as ellipsoidal heights were more difficult to obtain. In contrast, modern airborne campaigns observations are usually processed using gravity disturbances and ellipsoidal heights.

- Post-processing: depending on the campaign, the available input datasets may have already been postprocessed beforehand, meaning that one may be unable to reconstruct the raw observation data. This implies that inconsistencies made in the post-processing chains of different surveys are difficult to undo, especially since the metadata describing the processing strategies is often missing. The most common post- 
processing steps of the observations in the datasets are gridding and reduction to a reference surface (e.g. ellipsoids, offset-ellipsoids).

- Accuracy: the observation accuracy obtained by different campaigns can also be heterogeneous, as it depends on instrumentation, transportation of the instruments and the possibly inaccurate postprocessing.

- Spectral content: regarding the spectral content of the data one also might observe varying behavior, especially in the higher frequency domain. This can be attributed to multiple reasons. E.g., when dealing with airborne gravity observation one must keep in mind that due to flight dynamics and limitations of the instruments the raw measurement data needs to be lowpass filtered down to several $\mathrm{km}$ wavelength. Also, it is possible that during further postprocessing (e.g. gridding, collocation) the data may have already been spectrally limited.

As the aim of the AntGG grid is primarily to represent the available gravity field information, no in-depth evaluation or homogenization of these datasets has been performed so far. To tackle this circumstance and the fact that new gravity data is available by now the German Research Foundation (DFG) funded the so-called AntGrav project as part of which this study is performed. The main purpose of this project is to compute an optimally combined gravity model from satellite and ground data for the Antarctic continent. From this dataset a model of the bedrock topography shall then be derived by means of geophysical inversion.

For the targeted combination of gravity datasets reliable knowledge about the actual accuracy and spectral content of individual measurement campaigns is required as well as the possibility to detect larger outliers and systematic effects. Thus, an adequate evaluation method is needed, which is applicable on the wide range of different survey campaigns available in Antarctica. The method shall therefore meet the following criteria:

- Consistency: the method shall be able to inspect all different kinds of campaigns independently of their attributes and with unvarying evaluation quality.

- Independency: the evaluation method shall not be correlated to the actual measurements (subject of examination) allowing to make assessments about their correctness.

- Efficiency: as millions of datapoints need to be evaluated, the evaluation method shall be fast and efficient w.r.t. computation time and memory consumption.
- Correctness: obviously, the evaluation method shall be as correct as possible by itself.

The technique presented in this study will attempt to properly fulfill all these demands. To accomplish this, we make use of:

- a preexisting gravity field model: reducing gravity field information from a preexisting model and studying residuals is a feasible (and always applicable) method to examine gravity field observations. Such a gravity field model must be as independent and as correct as possible as well as offer the highest possible resolution. Further, it shall be given in the spectral domain in order to address the consistent adaption on different observation types (e.g. gravity anomalies and disturbances).

- a fast synthesis method: having gravity field information in the spectral domain, the transformation to the spatial domain (synthesis) is a time-consuming task when dealing with large scattered point datasets. To reduce computation time, it is proposed to apply a two-step synthesis method, where in a first step gravity field information is pre-calculated on regular geographic 3D grids and then in a second step interpolated on the individual scattered point positions.

In section 2 the used gravity field model SATOP1 is described in more detail, as it is generated specifically for the purpose of evaluation. Section 3 describes the synthesis method with all its characteristics. Subsequently, within section 4 the new method is applied to examine the AntGG grid. Finally, in section 5 examination results for two selected in-situ measurement campaigns are presented.

\section{The SATOP1 gravity field model}

As explained in section 1, an independent gravity field model shall constitute the basis of the evaluation method. To be truly independent, the model shall not contain any of the observations being evaluated. In the case of Antarctica, this means that no terrestrial gravity field information at all is to be included, because observations over Antarctica are generally sparse (and barely overlapping) and are target of the inspection itself.

Thus, the only available data sources left are satellite models as well as topographic forward models. Both sources show complementary spectral behavior: satellite models are very accurate in the low frequency domain but worsen with increasing resolution due to signal attenua- 
tion of the gravity field with altitude. Topographic models, on the other hand, do not include lateral density variations or isostatic effects, which leads to a decreased accuracy especially in the low frequency domain (cf. Fig. 1). Because of these complementary characteristics it is logical to use both data sources in a combined (SA)tellite-(TOP)ographic model (hence called SATOP1).

For the combined model SATOP1, the satellite model GOCO05s (Mayer-Gürr, 2015) and the EARTH2014 (Rexer et al., 2016) topographic model are chosen (up to d/o 5480), as both models include actual data sources and are based on mature processing techniques. As an optimal combination method, a variance-based stacking on normal equation level in the spectral domain is applied, similarly to the model SatGravRET14 (Hirt et al., 2016). This strategy can be interpreted as regularization of the satellite system with the topographic model as a-priori information. Mathematically, the combination can be described as:

$$
\underline{x}_{S A T O P 1}=C_{S A T O P 1}\left(\underline{q}_{G O C O 05 S}+\operatorname{diag}\left(\underline{v a r}_{E A R T H}^{-1}\right) \underline{x}_{E A R T H}\right)
$$

with

$$
C_{S A T O P 1}=\left[N_{G O C O 05 s}+\operatorname{diag}\left(\underline{v a r}_{E A R T H}^{-1}\right)\right]^{-1}
$$

where $N_{\text {GOCO05s }}$ is the normal equation matrix and $\underline{q}_{G O C O 05 s}$ is the right-hand side of the GOCO05s model, $\underline{x}_{E A R T H}$ represents the coefficients and $\underline{v a r} \underline{G A R T H}_{\text {the vari- }}$ ances of the EARTH2014 model, and finally, $\underline{x}_{S A T O P 1}$ describes the resulting SATOP1 model coefficients and $C_{S A T O P 1}$ the corresponding covariance matrix.

Within the processing of SATOP1, the choice of a realistic variance for EARTH2014 $\left(\underline{\text { var }}_{\text {EARTH }}\right)$ is crucial, as it controls the spectral transition from the satellite to the topographic model. Since topographic forward modelling as used in EARTH2014 does not provide any statistical measures for its result, one has to find a different method to derive model variances. For SATOP1 it is assumed that those variances are mostly degree-dependent and hence can be simplified to degree-variances (no order dependency). The degree-variances are then derived empirically from the comparison to another independent model. In this case, XGM2016 (Pail et al., 2018) is used:

$$
\operatorname{var}_{l}^{\text {EARTH }} \sim \sum_{m=-l}^{l} \frac{\left(c_{l m}^{E A R T H}-c_{l m}^{X G M}\right)^{2}}{2 l+1}
$$

and $c_{l m}^{X G M}$ denote the spectral coefficients of the EARTH2014 resp. XGM2016 models, with degree $l$ and order $m$ (negative orders indicate sine-coefficients). The spectral characteristics of the models and the empirical variances are visualized in Fig. 1a in form of gravity anomaly degree-RMS.

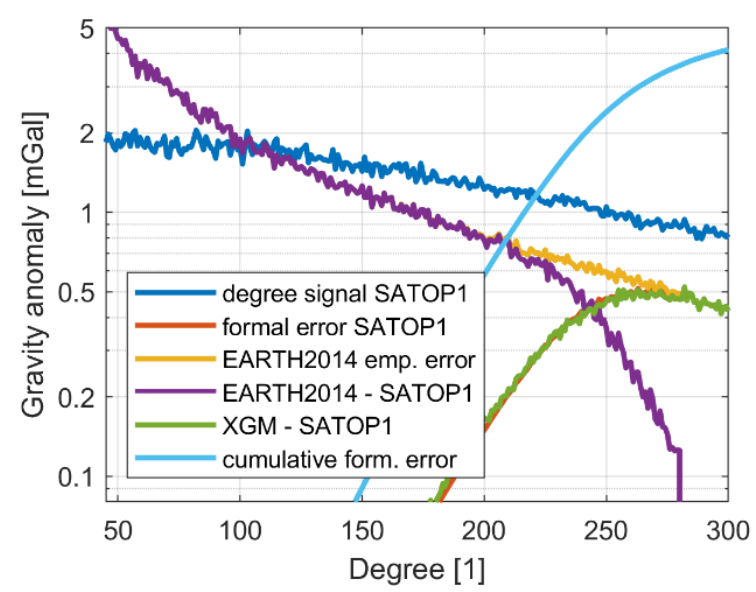

(a)

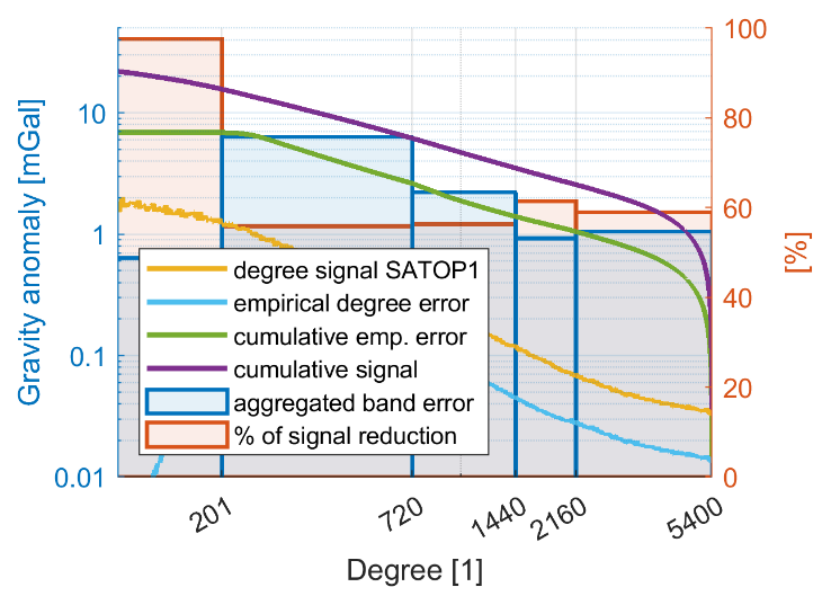

(b)

Figure 1: Spectral characteristics of SATOP1 (in spheroidal-harmonics at $4 \mathrm{~km}$ altitude): (a) degree-RMS of the satellite band in terms of gravity anomalies. Dark blue: signal of SATOP1, orange: formal error of SATOP1, yellow: empirical error EARTH2014 (for regularization), violet: difference EARTH2014-SATOP1, green: difference XGM2016SATOP1, light blue: SATOP1 cumulative formal error. (b) SATOP1 error estimate in terms of gravity anomalies. Yellow: signal of SATOP1, light blue: empirical error of SATOP1 (XGM2016-SATOP1), green: SATOP1 cumulative empirical error, violet: cumulative SATOP1 signal (unreduced), dark blue bars: aggregated SATOP1 errors within depicted bands, orange bars: percentage of SATOP1 band signal reduction (refers to right axis).

In contrast to preceding strategies (Hirt et al., 2016) the combination of SATOP1 is performed purely in a spheroidal-harmonic domain adopting the GRS80 reference system (Moritz, 1980, 2000) - all items in Eqs. (1), (2) and (3) are given w.r.t. this domain. To accomplish this, 
Jekeli's transform (Jekeli, 1981) is applied in a preceding step to transform all input quantities into the spheroidalharmonic domain. Performing the degree-variance-based combination within this domain is preferred, since the coefficient smearing (=correlation) between adjacent degrees (as implied by Jekeli's transform) on (or near) spheroidal surfaces is reduced. A second and perhaps more important advantage is, that due to the same reason, spectral band cuttings in the spheroidal domain do not lead to truncation artefacts as e.g. seen in EGM2008 (Pavlis et al.2012) when omitting its highest degrees (e.g. d/o 2160 upwards).

Thus, the investigation of different spectral bands in the spatial domain (on or near the spheroid) is reasonable. For the following synthesis six (spheroidal) spectral bands ([2-200], [201-359], [360-719], [720-1439], [1440-2159], [21605480]) are selected. The first limit is chosen, as it represents an empirical upper boundary for a (nearly) satelliteonly solution, the latter limits correspond to spatial resolutions commonly used in global gravity field modelling. To be compatible with the spherical harmonic definition of different gravity field functionals, the specific bands are truncated in the spheroidal harmonic domain and then transformed into the spherical harmonic domain (Jekeli, 1981) for an ordinary synthesis.

Error estimations of the different spectral bands (commission errors) are shown in Fig. 1b: they are derived in the same way as the formal errors of the EARTH2014 model by comparing SATOP1 against XGM2016 extended by EGM2008 from d/o 720 up to 2159. Due to lack of independent comparison data, errors above d/o 2159 are extrapolated. As expected, the largest contribution to the commission error (at a simulated flight altitude of $4 \mathrm{~km}$ ) occurs in the lowest topographic band (between d/o 201 and 719). While the estimated percentage of signal reduction stays widely stable (at about $60 \%$ ), the gravity field signal, and thus also the commission error, is attenuated with increasing $\mathrm{d} / \mathrm{o}$.

\section{The AGRID3D synthesis method}

Performing a spherical harmonic synthesis up to d/o 5480 is a CPU-intensive task, especially when dealing with scattered points, as Legendre polynomials need to be recalculated for every single observation. As pointed out in the introduction, a two-step synthesis may be better suited for the purpose of evaluating big data volumes.

Firstly, it exploits the advantage of regular grid synthesis, where Legendre polynomials are only evaluated in- dividually for different latitudes, but not for every single point (Sneeuw 1994). Thus, a geographic grid has to be chosen as a base grid, because polar stereographic grids, for instance, would feature varying latitudes for each grid point.

Secondly, due to the fact that the minimal wavelength is known to be limited (d/o 5480 corresponding to 2' spatial resolution), a 2-times oversampled grid is also known to give good interpolation results, as it fulfills the NyquistShannon sampling theorem (Shannon 1949). Having a precalculated grid at hand, the computation time of the synthesis problem scales linearly w.r.t. the number of points being interpolated and is in contrast to the rigorous synthesis (nearly) independent of the maximum d/o (i.e. the number of support points).

Even though other methods exist for the upward continuation of the gravity field besides 3D interpolation (e.g., Rapp (1997), Ivanov et al. (2018)), this method is favourable as it is simple, robust, very fast, and the accuracy of the result is independent of the distance to the surface (cf. subsection 3.2).

\subsection{Properties and components of AGRID3D}

The first step of the two-step synthesis is the calculation of the regular 3D grid. The dimension of the grid has to be chosen in a way which ensures that every point to be validated is located safely within the grid boundaries. The final AGRID3D properties can be summarized as follows (cf. Fig. 2):

- Grid extent: in longitudinal direction the grid ranges from $0^{\circ}$ to $360^{\circ}$, in latitudinal direction from $-60^{\circ}$ to $-90^{\circ}$, and in vertical direction from $-1 \mathrm{~km}$ up to $6 \mathrm{~km}$. On every periodic limit, the grid was extended by 5 cells to avoid increasing interpolation errors near the boundaries.

- Grid resolution: in order to ensure that the spherical grid is oversampled a least twice, a latitudinal sampling of 1 ' is chosen. Adapting to meridian convergence, the longitudinal sampling is reduced to 2 ' at $-60^{\circ}$ lat. and $4^{\prime}$ from $\sim-75.4^{\circ}$ southwards. This reduces the grid size and hence saves memory space. For the vertical component, a constant spacing of $200 \mathrm{~m}$ is selected empirically.

- Spectral bands: to enable also a spectral evaluation, 6 distinct grids are calculated for the different spectral bands as defined in section 2 .

- Gravity field functionals: as all observation data of the AntGG dataset (Scheinert et al. 2016) are given either in the form of gravity anomalies or gravity distur- 
bances, dedicated sets of grids are calculated for these two functionals.

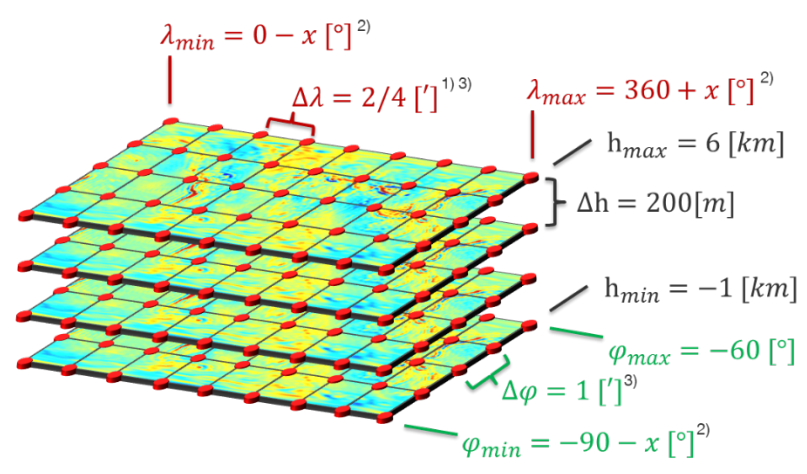

Figure 2: Visualization of the grid properties of the AGRID3D dataset. Notes: 1. Meridian convergence is considered. 2. Limits are extended to simulated periodicity. 3. 2x Nyquist-frequency (for d/o 5400) is guaranteed.

One complete $3 \mathrm{D}$-grid (from $-60^{\circ}$ to $-90^{\circ}$ ) has therefore a memory size of $\sim 4.1 \mathrm{~GB}$ (in double precision). This marks also the recommended minimum RAM requirement of a system to perform an interpolation based on AGRID3D. Since different grids are needed for the spectral bands $(6 \mathrm{x})$ and gravity field functional (2x), the overall size of the complete AGRID3D dataset is $\sim 49 \mathrm{~GB}$.

The computation of the grid itself is performed on resources of the Leibniz Computing Center (LRZ). For the stable calculation of the Legendre polynomials, methods based on extended numbers (Fukushima, 2012) are used.

\subsection{Interpolation performance}

Based on the resulting AGRID3D dataset, the gravity values at arbitrary points within the extent of the grid are obtained by interpolation. Within this study, a common (tri-)cubic interpolation approach (Lekien and Marsden, 2005) is used, as it shows good performance (cf. Fig. 4) while keeping memory requirements and calculation costs low. Before those interpolated points can be used as evaluation reference, it must be verified that the interpolation method is accurate and in agreement with a rigorous direct scattered point synthesis.

To prove the correctness of the interpolation method itself (as required for the purpose of evaluation), it is proposed to simulate a worst-case scenario, where all interpolated points are located at the maximum distance cen- tered between the support points (cf. Fig. 3a). This leads to a validation grid which is shifted by half the grid spacing in all three directions relatively to AGRID3D. This validation grid is then evaluated with two different strategies: first, the SATOP1 model is synthesized rigorously to get reference values (using all bands, $d / o$ 2-5480). Second, the interpolation based on AGRID3D is applied to obtain homologous values. Latter values are not only obtained using the cubic approach, but also involving the widely used linear and spline interpolation methods, enabling comparisons between the different techniques. As the first strategy is assumed to be error-free, differences in the values of both methods represent the interpolation error.

Statistical evaluations of those differences for different height layers and interpolation approaches are shown in Fig. 4, and an example for the spatial distribution of the cubic interpolation errors on a near-surface height layer of $100 \mathrm{~m}$ is shown in Fig. 3b for the whole spectrum (d/o 2-5480). Generally, a strong correlation of the interpolation error with high-frequency signals (e.g. due to mountains) is evident. This implies that the interpolation error is also strongly correlated with height, since upward continuation of the gravity field leads to an attenuation of higher frequencies and thus reduced interpolation errors. Empirically, this fact can also be seen in the statistics (cf. Fig. 4). Although the spline-interpolation method shows the best interpolation accuracy, the cubic approach is more favorable, as the actual implementation requires less memory and the performance is still within a reasonable limit for the purpose of evaluation (as uncertainties within the SATOP1 model are expected to exceed the interpolation error).

\section{AntGG inspection}

In a first application, the evaluation method is used to inspect the AntGG (Scheinert et al., 2016) grid. The AntGG dataset consists of a polar stereographic regular surface grid (ice layer including) of gravity anomalies with a spacing of $10 \mathrm{~km}$. The AntGG gravity anomalies are further spectrally limited by an average operator to minimize aliasing effects due to the grid sampling.

\subsection{Reducing the SATOP1 model}

The SATOP1 model is interpolated on the AntGG grid points (using the cubic AGRID3D interpolation, see section 3) for increasing spectral content (using the 6 bands 


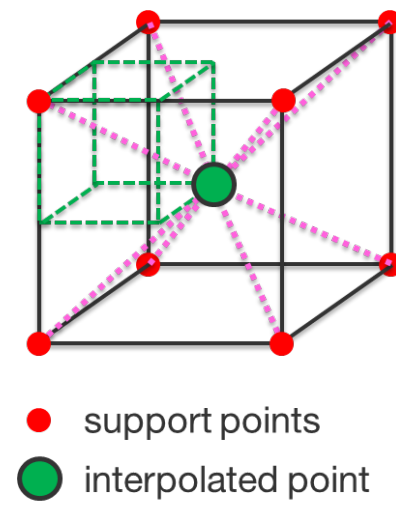

(a)

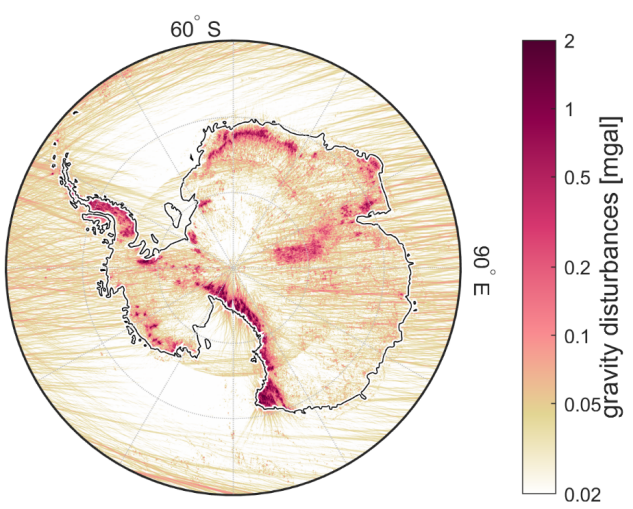

(b)

Figure 3: (a) Visualization of the validation concept. Interpolation grid points (shown in green) lie centered between support grid points (shown in red). This is clarified by dashed green lines. The chosen setup leads to equal maximum distances of the interpolation point to all surrounding support points (indicated by dashed purple lines). (b) Interpolation errors (in terms of gravity disturbances) for the cubic interpolation approach on a $100 \mathrm{~m}$ height layer for the complete SATOP1 model (including all spectral bands).

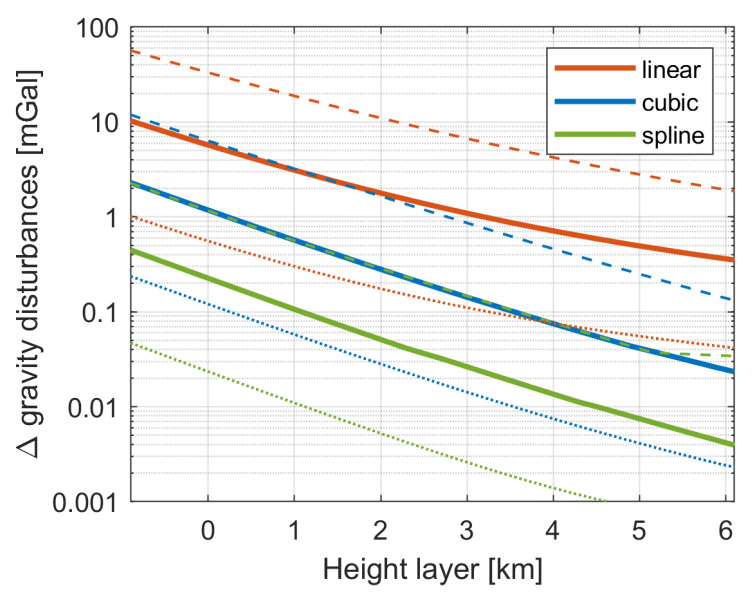

Figure 4: Statistical characteristics of the AGRID3D worst-case scenario errors for different heights and interpolation strategies. Shown are RMS (dotted), absolute maximum (dashed) and absolute $99.9 \%$ quantile (continuous) for the linear (red), cubic (blue) and spline (green) interpolation method. Using the cubic approach, on $100 \mathrm{~m}$ height, $99.9 \%$ of all values are within $\pm 2 \mathrm{mGal}$, on $2 \mathrm{~km}$ all values are within this range.

as defined in section 2). The resulting grids are then subtracted from the original AntGG grid to obtain residuals. As the sources of the gravity data and the SATOP1 model are assumed to be mostly independent, it is reasonable to use these residuals for the purpose of cross-validation: if they are small in a certain region, this is a strong indicator that both datasets are correct (meaning that they are error-free and encompass the same spectral content). Vice versa, when residuals become larger, it should be kept in mind that in principle no statement of the error source can be made. The difference could either result from errors in the real observations, or from inadequacies within the SATOP1 model. However, the analysis of residuals may still help finding suspicious data, while simultaneously reliably identifying trustworthy data. Further, these residuals can also be used to estimate the spectral content of AntGG by inspecting statistical properties for different band reductions (cf. Fig. 5a).

Looking at the remaining standard deviation of the reductions (cf. Fig. 5a), one can identify a minimum when using SATOP1 up to $d / o$ 719. Thus, it is deduced that the spectral content of the AntGG grid is limited at approx. this resolution. In fact, the AntGG grid is low-pass filtered to reduce aliasing effects on the $10 \mathrm{~km}$ spaced grid. Hence, the $d / o 719$ reduction is concluded to fit best to the AntGG data and is therefore chosen for further investigations. The empirical covariances (cf. subsection 5.2) also support this choice, although some longer distance patterns remain due to discrepancies in the models (e.g. isostatic effects or incomplete terrain data in SATOP1, errors in the AntGG grid).

\subsection{AntGG validation}

Reductions using SATOP1 also include the error of the topographic EARTH2014 model and therefore cannot be directly used to validate other datasets. Nevertheless, SATOP1 also consists of the high-accuracy satellite model part up to about d/o 200 (cf. Fig. 1). 


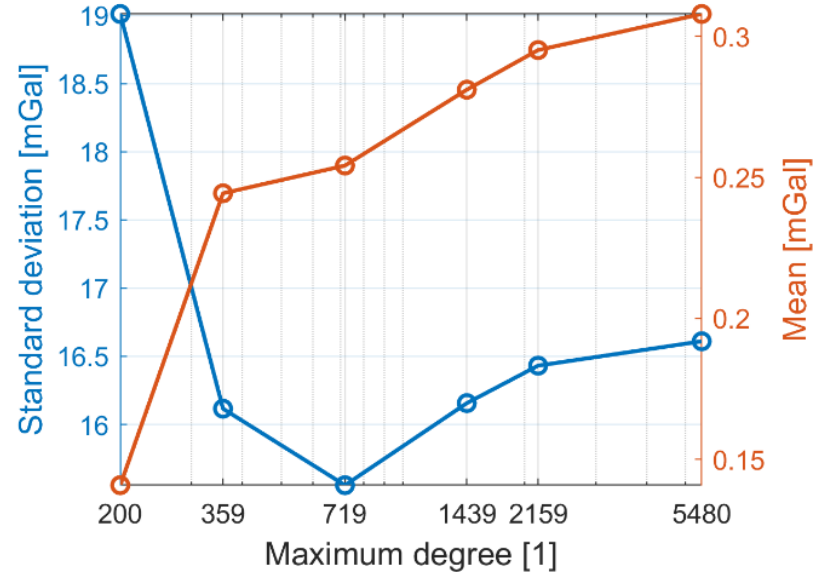

(a)

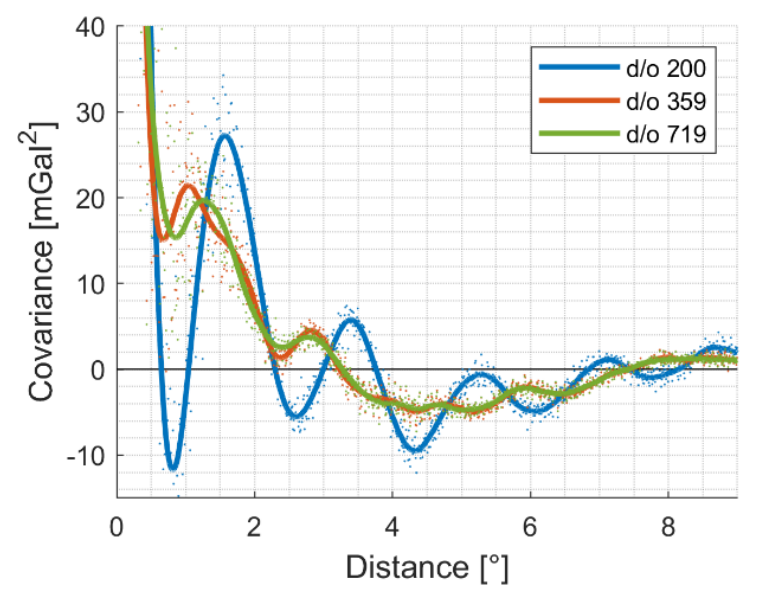

(b)

Figure 5: Statistical properties of the AntGG-SATOP1 inspection: (a) Standard deviation (blue) and mean (red) for varying maximum degree of the SATOP1 model reduction from the AntGG grid. (b) Empirical covariances depending on the spherical distance for different maximum degree reductions (200-359-719). Colored dots indicate the scattering of the smoothed covariance functions (continuous lines).

This issue can be exploited when applying a low-pass filtering to the data which attenuates gravity field information above $\mathrm{d} / \mathrm{o} 200$. As the spectral truncation of the signal in the frequency-domain is obviously not (easily) possible (since the data is only available in the spatial domain), Gaussian filtering (Jekeli, 1981) is applied instead to widely reduce the influence of the topographic model.

The spectral strength of the Gaussian low-pass filtering is determined by the choice of the full-width-halfmaximum (FWHM) distance (cf. Fig. 7a). For a reliable reduction of signals beyond d/o 200 a distance of $80 \mathrm{~km}$ is chosen. For a less reliable but higher resolution result a

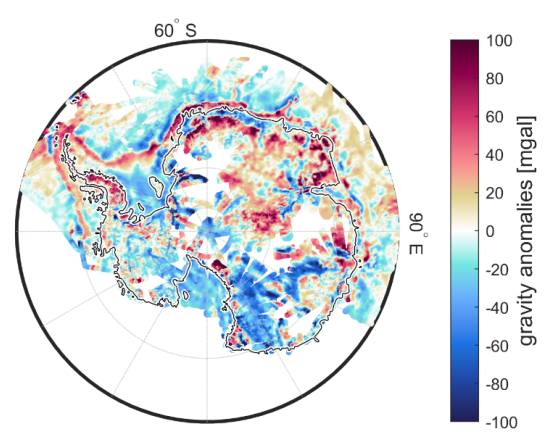

(a)

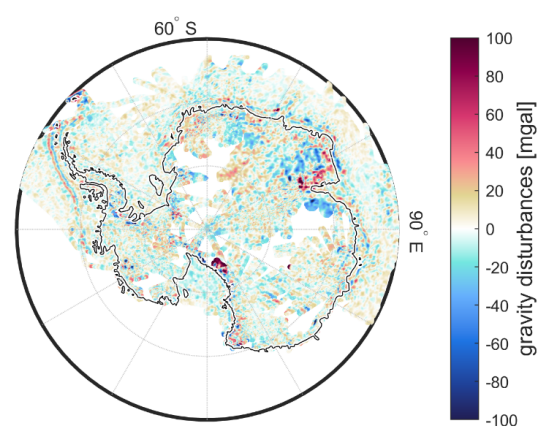

(b)

Figure 6: (a) The AntGG grid as is with white areas where no data is available. (b) Difference to SATOP1, limited to d/o 719.

second variant is calculated with $40 \mathrm{~km}$ FWHM. As an example, the $80 \mathrm{~km}$ FWHM filtered reduction is shown in Fig. 7b: signals within this plot arise with high certainty from errors in the AntGG datasets.

Within the Gaussian filtering, the occurring filter uncertainties near data gaps are considered by weighting down the signal in those regions. The filter uncertainty itself is estimated through comparison of the complete with the incomplete grid filtering result of SATOP1 data.

In a next step the low-pass filtered reductions $(\Delta f)$ can be further processed to derive a final error measure. To achieve this, local standard deviations $(\bar{s})$ are derived applying weighted integrals once again using a Gaussian kernel $\left(W_{G}\right)$. The appropriate formula can be summarized as (Jekeli, 1981):

$\bar{s}(\theta, \lambda):=\sqrt{\left(W_{G}{ }^{\star} \Delta f^{2}\right)(\theta, \lambda)}=\sqrt{\sum_{i=1}^{n} W_{G}\left(\psi_{i}, d_{F W H M}\right) \Delta f_{i}^{2}}$

with the spherical distance:

$$
\cos \psi_{i}=\cos \theta \cos \theta_{i}+\sin \theta \sin \theta_{i} \cos \left(\lambda-\lambda_{i}\right)
$$




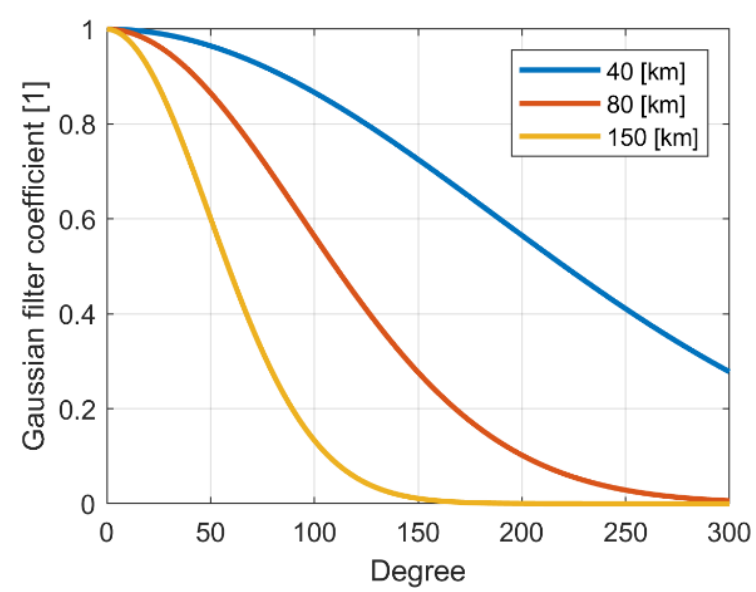

(a)

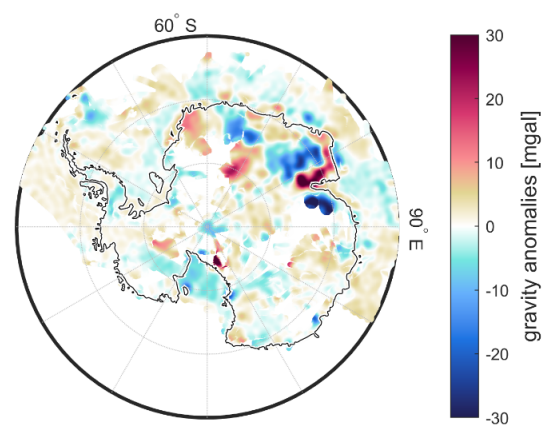

(b)

Figure 7: (a) The impact of different FWHM filter distances (40 km blue, $80 \mathrm{~km}$ red, $150 \mathrm{~km}$ yellow) in the spectral domain, depending on the spherical degree. (b) Difference AntGG-SATOP, up to d/o 719 (cf. Fig. 6b), 80 km FWHM Gaussian filtered.

As a suitable FWHM distance $\left(d_{F W H M}\right)$ for the Gaussian kernel the same as for the low-pass filtering is chosen. The final results are shown in Fig. 8a (for $80 \mathrm{~km}$ FHWM) and Fig. $8 \mathrm{~b}$ (for $40 \mathrm{~km}$ ). The latter may still contain some signals from the topographic model but also delivers a higher resolution than the $80 \mathrm{~km}$ version.

Having these error estimates at hand, one may now take an in-depth look into suspicious regions that show larger deviations by inspecting the underlying gravity observation campaigns (see section 5)

\section{In-situ evaluations}

As mentioned in section 1 , the presented examination strategy is not only meant for application to already combined and gridded datasets such as AntGG, but also to in-

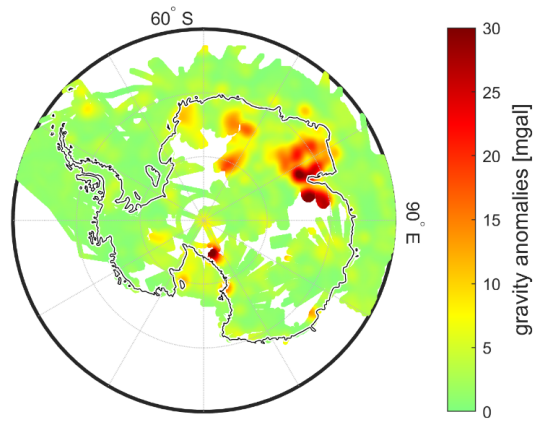

(a)

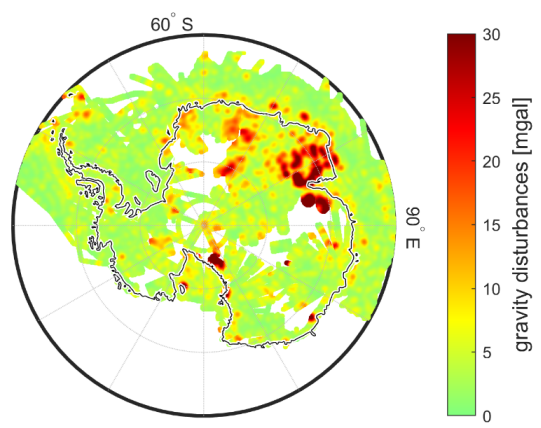

(b)

Figure 8: AntGG error estimate (standard deviation) based on (a) $80 \mathrm{~km}$ resp. (b) $40 \mathrm{~km}$ FWHM low-pass filtering.

situ measurements from various gravity campaigns (as the ones used in the AntGG grid). This has the advantage that possible errors/outliers may be identified on observation level and therefore eliminated/corrected without influencing other measurements. To showcase the application, two different campaign types are selected: firstly, a larger recent airborne survey campaign named AGAP (Ferraccioli et al., 2011) and secondly, an older ground measurement campaign of the Antarctic Peninsula, named BAS-LAND (Renner et al. 1985).

\subsection{AGAP dataset}

Within the Antarctica's Gamburtsev Province (AGAP) project, a comprehensive airborne gravity map of the Gamburtsev Subglacial Mountains was recorded during the 2008/09 field season with a line spacing of $5 \mathrm{~km}$ and tieline interval of $\sim 33 \mathrm{~km}$ at a mean altitude of $\sim 4 \mathrm{~km}$. The whole dataset consists of over 2 million datapoints of gravity disturbances.

Performing the same reductions as in subsection 3.1 (but now in-situ, on the actual point of measurement), 


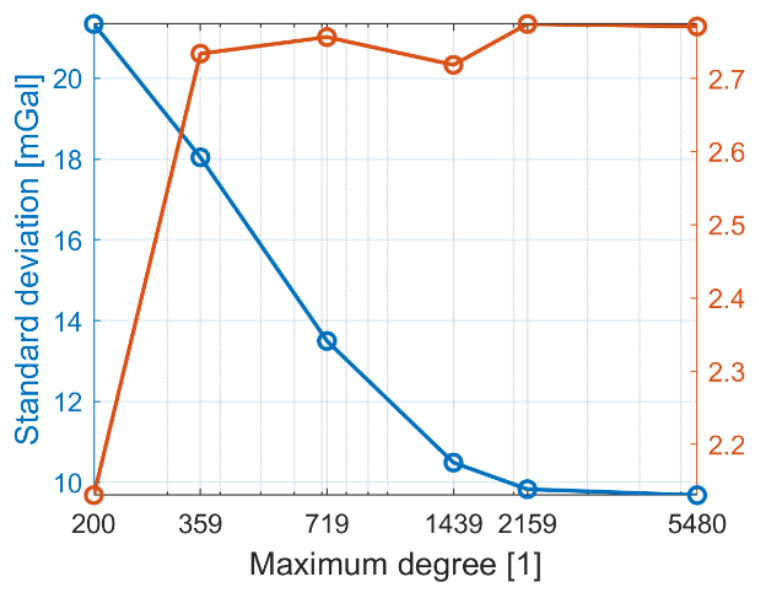

(a)

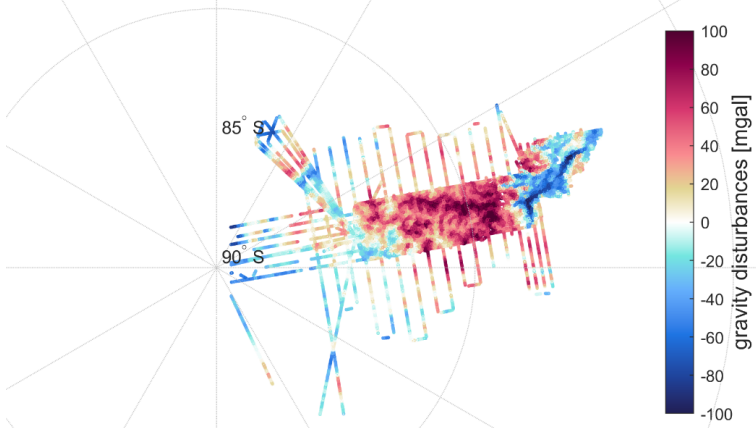

(c)

Figure 9: (a) Standard deviation (blue) and mean (red) for varying maximum degree of the SATOP1 model reduction from the AGAP observations. (b) Empirical covariances depending on the spherical distance for different maximum degree reductions (200-359-719-5400). (c) The AGAP observations as provided. (d) Differences to SATOP1 up to d/o 5400.

the results are shown in Figs. 9a-d: Looking at the statistics (Figs. 9a-b), the reduction performance can be considered outstanding while keeping in mind that from d/o 200 upward only topographic forward modelling is used. This also suggests a lack of higher frequency isostasy (beyond $\mathrm{d} / \mathrm{o} 200$ ) in this region (maybe due to the glacial coating). The covariances (Fig. 9b) show an expected behavior: the steepness towards the first minimum increases and the oscillation around 0 decreases with a higher reduction degree.

A visual inspection of the dataset (Figs. 9c-d) reveals no obvious errors or outliers. Also, the detected offset of $2 \mathrm{mGal}$ (Fig. 9a) meets the expectations. Thus, it can be summarized that the AGAP data is most probably very trustworthy. Further, it can be seen (Fig. 9a and visually in 9c) that the spectral content is not significantly limited below d/o 2159 (e.g. due to filtering of airborne data).

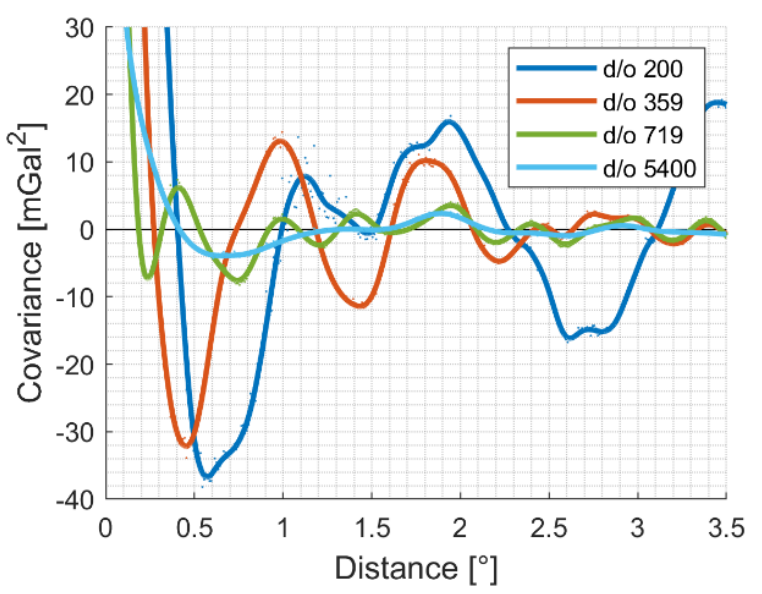

(b)

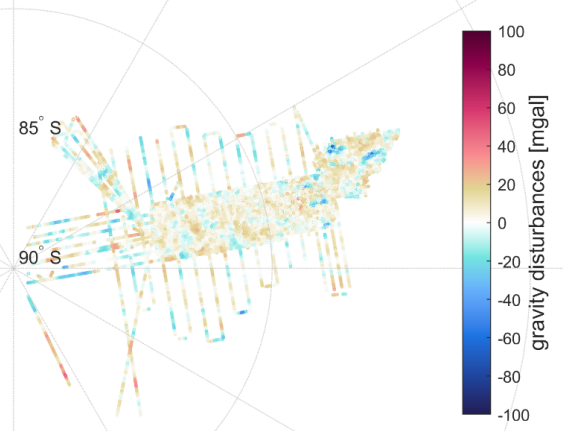

(d)

It is worth mentioning that due to the use of AGRID3D in combination with the cubic interpolation approach, the reduction of the 2 million observation points can be calculated within a second on a standard PC.

\subsection{BAS-LAND}

In contrast to the AGAP campaign, the BAS-LAND dataset contains ground measurements of the Antarctic Peninsula (cf. Fig. 10c) from the pre-GNSS era. This leads to an introduction of some new error sources.

Firstly, the geolocalization of points is probably less accurate especially in the vertical direction, as this coordinate refers to an orthometric height. Consequently, the observations are given in terms of gravity anomalies. For the reduction with AGRID3D, a (quasi-)geoid model is therefore needed to convert the physical coordinates 
to geometric ones. To maintain consistency, this (quasi)geoid is calculated correspondingly also from the SATOP1 model, introducing on top of all other effects its own (height-)uncertainty.

Further, terrestrial observations in general include more higher-frequency content than airborne measurements, as no upward continuation is embedded. For the airborne data it can be assumed that due to the common flight altitudes not much signal above d/o 5400 is left. This may not be true for terrestrial measurements, especially in mountainous regions (as the Antarctic Peninsula). So, even if the SATOP1 model were totally correct, there would still be gravity information above $\mathrm{d} / \mathrm{o} 5400$ that is not covered by the model.

Another aspect to consider, altough the effect is considered to be minor, is the possible temporal variability of the gravity field signal, as the BAS-LAND data was recorded about 30 years prior to the SATOP1 data. Variable ice-thicknesses may introduce changes in the gravity field above mGal-level, as $25 \mathrm{~m}$ ice thickness corresponds to roughly $1 \mathrm{mGal}$ Bouguer gravity (without considering glacial isostatic rebound, variable ice densities etc.).

Finally, the overall consistency of the data may be not as good as from airborne campaigns since e.g. uncertainties due to drift effects of relative gravimeters increase with time and transportation conditions, two influence factors that are generally unknown for many terrestrial datasets (even though Renner et al. 1985 provide some hints for the actual campaign).

When looking at the statistics of the residuals after reducing SATOP1 (Fig. 10a), it can be seen that the standard deviation is twice as high as in the AGAP dataset. This is not surprising regarding all the additional error sources mentioned above. Nevertheless, the behavior of the empirical covariances (Fig. 10b, first minimum positive) implies some systematic effects. These effects can also be found in Fig. 10d as longer wavelength effect (positive in the mountains, negative over the ocean). As there could be different reasons for this phenomenon, no clear assertion can be made in this case. A common cause for such effects in terrestrial observations may be the systematics introduced through the choice of the measurement site (e.g. measuring through valleys, not over crests). The fact that with increasing reduction degree the effect decreases (cf. Fig. 10b) supports this theory (as unilateral measuring can be interpreted as source for aliasing).

Beside the longer-wavelength pattern, a strongly negative measurement track (marked in Fig. 10d) can be identified. As there is no obvious reason in this case (e.g. higher mountains) and the cut in the track is abrupt, a gross error in the observations seems reasonable. The presence of the higher mean value (Fig. 10a) of over $20 \mathrm{mGal}$ is not unusual for a campaign dating back to a time when the tie to global gravity reference systems was not easy to establish (especially in Antarctica).

\section{Conclusions}

It is shown (through section 3b, 4 and 5) that the presented evaluation approach widely fulfills the demands stated in section 1 . Thus, the method is fit for application to further datasets, with the final objective to inspect all datasets included in the AntGG database as well as all other campaign data available. The examples in section 5 were chosen to be most differing in terms of campaign type and therefore represent the majority of the available datasets. Thus, individual campaign examinations will be performed, accordingly.

After the evaluation and correction phase of the data is finished, the SATOP1 reductions can be reused for the remove-compute-restore procedure within the least squares collocation approach when producing the final regional gravity field solution. This is possible since the SATOP1 reductions rely on a spectral model and thus can be adapted consistently to all different kinds of gravity field functionals on arbitrary points in space.

Further, with the SATOP1 model (section 2) two innovations are introduced: firstly, the regularization of the satellite model was performed based on spheroidal harmonic degree variances, minimizing errors due to an implicit downward continuation (towards the poles). Secondly, spectral bands were cut out also in the spheroidal domain in order to avoid truncation effects (in contrast to band limitations in the spherical domain).

In summary, it can be concluded that the overall evaluation performance of the presented approach strongly depends on the dataset at hand - sparsely distributed terrestrial datasets are more difficult to inspect, as they generally feature a lower spatial density while their spectral content is higher (in comparison to airborne observations). This inevitably leads to more aliasing, preventing assessments on small scales (e.g. single points) and possibly also on larger scales (although gross errors may still be detectable, c.f. subsection 5.2). Dense airborne datasets on the other hand are easier to validate for the same reason, even though there is the additional difficulty with the (mostly unknown) low-pass filtering embedded in the data.

Finally, it is important to mention that neither the gravity field model nor the synthesis method presented 


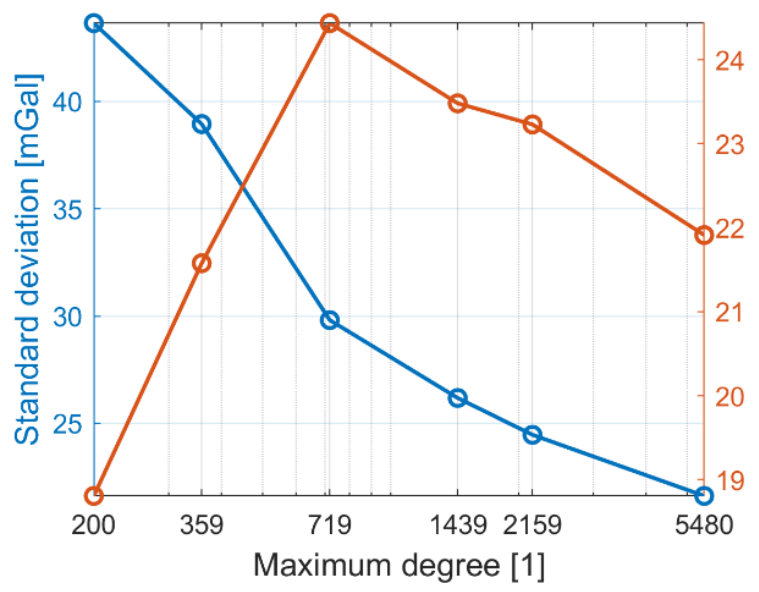

(a)

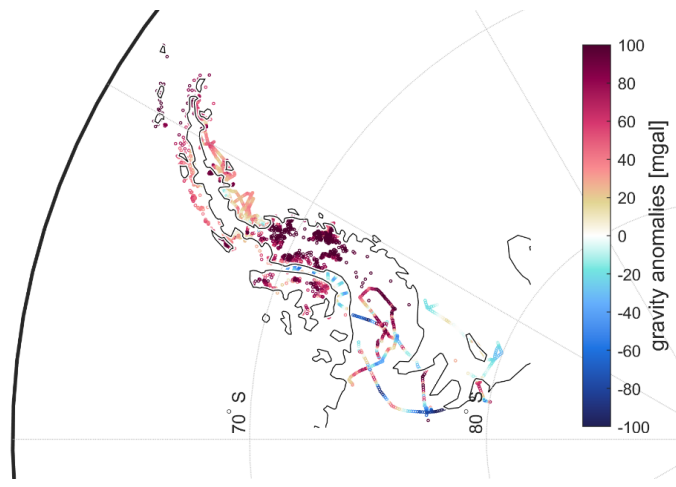

(c)

Figure 10: (a) Standard deviation (blue) and mean (red) for varying maximum degree of the SATOP1 model reduction from the BAS-LAND observations. (b) Empirical covariances depending on the spherical distance for different maximum degree reductions (200-359-719-5400). (c) The BAS-LAND observations as provided. (d) Differences to SATOP1 up to d/o 5400, mean value subtracted.

is restricted to the Antarctic region; one is free to use this evaluation strategy globally for nearly all gravity field functionals/observations. The application might be reasonable especially in other sparsely surveyed regions (e.g. Africa or parts of Asia).

Acknowledgements: This work was supported by the Deutsche Forschungsgemeinschaft (DFG) in the framework of the priority programme "Antarctic Research with comparative investigations in Arctic ice areas" by the grants PA 1543/18-1 and SCHE 1426/24-1.

\section{References}

Ferraccioli F., Finn C. A., Jordan T. A., Bell R. E., Anderson L. M. and Damaske D, 2011, East Antarctic rifting triggers uplift of the Gamburtsev Mountains, Nature, 479, 388-392. doi:10.1038/nature10566
Fukushima T., 2012, Numerical computation of spherical harmonics of arbitrary degree and order by extending exponent of floating point numbers, J. Geod., 86, 271-285. doi:10.1007/s00190-011-0519-2

Hirt C., Rexer M., Scheinert M., Pail R., Claessens S. and Holmes S., 2016, A new degree-2190 (10 km resolution) gravity field model for Antarctica developed from GRACE, GOCE and Bedmap2 data., J. Geod., 90, 105-127.

Ivanov K. G., Pavlis N. K. and Petrushev P., 2018, Precise and efficient evaluation of gravimetric quantities at arbitrarily scattered points in space, J. Geod., 92, 779-796. doi:10.1007/s00190-017-1094-y

Jekeli C., 1981, Alternative methods to smooth the Earth's gravity field, retrieved from http://adsabs.harvard.edu/abs/1981amse. book

Lekien F. and Marsden J., 2005, Tricubic interpolation in three dimensions, Int. J. Numer. Methods Eng., 63, 455-471. doi: $10.1002 /$ nme.1296

Mayer-Gürr T., 2015, The combined satellite gravity field model GOC005s, EGU General Assembly Conference Abstracts, 17, p. 12364.

Moritz H., 1980, Geodetic reference system 1980, Bulletin Geodesique, 54, 395-405. doi:10.1007/BF02521480 
Moritz H., 2000, Geodetic Reference System 1980, J. Geod., 74, 128. doi:10.1007/s001900050278

Pail, R. Fecher T., Barnes D., Factor J. F., Holmes S. A., Gruber T. and Zingerle P., 2018, Short note: the experimental geopotential model XGM2016, J. Geod., 92, 443. doi:10.1007/s00190-017-10706

Rapp R. H., 1997, Use of potential coefficient models for geoid undulation determinations using a spherical harmonic representation of the height anomaly/geoid undulation difference, J. Geod., 71, 282-289. doi:10.1007/s001900050096

Rexer M., Hirt, C. Claessens, S. and Tenzer R., 2016, Layer-Based Modelling of the Earth's Gravitational Potential up to 10-km Scale in Spherical Harmonics in Spherical and Ellipsoidal Approximation, Surv. Geophys., 37, 1035. doi:10.1007/s10712-016-9382-2
Scheinert M., Ferraccioli,F., Schwabe J., Bell R., Studinger M., Damaske D., Jokat W., Aleshkova N., Jordan T., Leitchenkov Blankenship G. D. D., Damiani T. M., Young D., Cochran J. R., Richter T. D., 2016, New Antarctic gravity anomaly grid for enhanced geodetic and geophysical studies in Antarctica, Geophys. Res. Lett., 43, 600-610.

Shannon C. E., 1949, Communication in the Presence of Noise, IEEE Proceedings, 37, 10-21. doi:10.1109/JPROC.1998.659497

Sneeuw N., 1994, Global spherical harmonic analysis by leastsquares and numerical quadrature methods in historical perspective, Geophys. J. Int., 118, 707-716. 